\title{
Disparate Mechanisms for Hypoxic Cell Injury in Different Nephron Segments Studies in the Isolated Perfused Rat Kidney
}

\author{
M. Brezis, P. Shanley, P. Silva, K. Spokes, S. Lear, F. H. Epstein, and S. Rosen \\ Charles A. Dana Research Institute, Harvard-Thorndike Laboratory of Beth Israel Hospital, Departments of Medicine and Pathology, \\ Harvard Medical School and Beth Israel Hospital, Boston, Massachusetts 02215
}

\begin{abstract}
Hypoxic injury was evaluated morphologically in the proximal tubule and in the medullary thick ascending limb of isolated rat kidneys perfused for 90 min without $\mathrm{O}_{2}$ or with various metabolic inhibitors. Inhibition of mitochondrial respiration (with rotenone, antimycin, oligomycin) or of intermediary metabolism (with monofluoroacetate, malonate, 2-deoxyglucose) caused reduction in renal oxygen consumption, renal function, and ATP content comparable with those elicited by oxygen deprivation. Metabolic inhibition produced hypoxiclike injury in the first portions of the proximal tubule, S1 and S2 ("clubbing" of microvilli, mitochondrial swelling), and the extent of damage was correlated with the degree of ATP depletion. In the third portion of the proximal tubule, S3, hypoxiclike damage (cytoplasmic edema or fragmentation) occurred most consistently when both aerobic and anaerobic metabolism were inhibited simultaneously. In the medullary thick ascending limb, none of the metabolic or mitochondrial inhibitors used could reproduce the injury of oxygen deprivation. Thus, the proximal tubule and the thick ascending limb have markedly different responses to cellular energy depletion, suggesting disparate mechanisms for hypoxic injury along the nephron.
\end{abstract}

\section{Introduction}

In anoxic cell injury, certain common pathways leading to cell death have been proposed, including calcium influx, lipid peroxidation, and energy depletion $(1,2)$. Cell types, however, differ in their response to $\mathrm{O}_{2}$ deprivation; for example, fibroblasts withstand anoxia better than neurons or renal epithelium (3). It is generally assumed that these variations are related to differences in cellular metabolic rate. In the kidney, different segments of the nephron exhibit different metabolic rates as well as different types of metabolism (4). Not only is there variation in the rate of metabolism along the nephron, but because of the architectural organization of the organ there is a corticomedullary gradient for oxygen with high oxygen tension in the cortex and very low oxygen tension, approaching critical levels, in the medulla (5). To determine whether tissue damage induced by anoxia in the kidney is related to energy depletion, we compared the effects of hypoxia with those of inhibitors of aerobic and/or anaerobic metabolism in different segments of the proximal tubule

Dr. Brezis is presently at the Department of Medicine, Hadassah University Hospital, Mount Scopus, Jerusalem, Israel. 1985.

Received for publication 21 March 1985 and in revised form 17 May

J. Clin. Invest.

(C) The American Society for Clinical Investigation, Inc.

$0021-9738 / 85 / 11 / 1796 / 11 \$ \$ 1.00$

Volume 76, November 1985, 1796-1806 as well as in the medullary thick ascending limb. The results indicate that these segments differ markedly in their response to energy depletion, suggesting disparate mechanisms for anoxic injury along the nephron.

\section{Methods}

Male Sprague-Dawley rats, weighing $310-470 \mathrm{~g}$, fed on Purina rat chow (Ralston Purina Co., Chicago, IL) and allowed free access to water, were used for all experiments. Perfusion of the right kidney was performed according to the technique described by Ross et al. (6). Regular perfusion medium, as used in control rats, consisted of a Krebs-Ringer's-Henseleit solution with bovine serum albumin at a concentration of $6.7 \mathrm{~g} / 100 \mathrm{ml}$ and glucose at $5 \mathrm{mM}$, gassed with $5 \% \mathrm{CO}_{2}, 95 \% \mathrm{O}_{2}$. Unless specified otherwise, kidneys were perfused for $90 \mathrm{~min}$ before their fixation for histological evaluation.

\section{Experimental groups}

(A) Control group $(n=17)$. Kidneys were perfused with regularly oxygenated perfusion medium. Under these conditions, the arterial $\mathrm{pO}_{2}$ was $468.9 \pm 8.6 \mathrm{mmHg}$ (mean $\pm \mathrm{SE}$ ). The data from an earlier group of control kidneys $(n=7)$ obtained at the beginning of the present study were found to be the same as those obtained in a newer group of control kidneys $(n=10)$ toward the end of the study $\sim 2$ yr later. These results were pooled into a single control group unless specified otherwise.

(B) Hypoxic perfusions $(n=12)$. Kidneys were perfused with regular perfusion medium that was gassed with $5 \% \mathrm{CO}_{2}, 95 \% \mathrm{~N}_{2}(n=7)$. Under these conditions, the arterial $\mathrm{pO}_{2}$ was $36.5 \pm 1.6 \mathrm{mmHg}$ (mean $\pm \mathrm{SE}$ ). In another set of experiments $(n=5)$ designed to produce histotoxic anoxia, regular oxygenation was used but the perfusion medium was supplemented with potassium cyanide $(\mathrm{KCN})$ at a final concentration of 2.5 $\mathrm{mM}$. Experimental groups C-E were designed to characterize the differential responses of nephron segments to mitochondrial/metabolic inhibition.

(C) Inhibitors of mitochondrial respiratory chain $(n=19)$. Kidneys were perfused with regular perfusion medium (gassed with $5 \% \mathrm{CO}_{2}, 95 \%$ $\mathrm{O}_{2}$ ) supplemented with one of the following inhibitors of electron mitochondrial transport: (1) rotenone, $10^{-5} \mathrm{M}(n=6) ;(2)$ antimycin, $10^{-5}$ $\mathrm{M}(n=6)$; (3) oligomycin, $10^{-5}-10^{-4} \mathrm{M}(n=7)$. These compounds were purchased from Sigma Chemical Co. (St. Louis, MO) and dissolved in absolute ethanol on the day of their use.

(D) Inhibitors of intermediary metabolism $(n=30)$. Kidneys were perfused with regularly oxygenated medium that was supplemented with one of the following inhibitors of intermediary metabolism: (1) sodium monofluoroacetate (MFAA) $5 \mathrm{mM}(n=6)$; (2) sodium malonate, 25$50 \mathrm{mM}(n=5)$; (3) 2-deoxyglucose (2DG), $50 \mathrm{mM}(n=10)$. In six of the experiments with 2DG, glucose was omitted from the medium. Since the results, with or without glucose, did not differ, the two subgroups were pooled.

To test for the specificity of the effect obtained with metabolic blockade, reversal was sought with the addition of a substrate that would bypass the inhibition of aconitase by monofluoroacetate, as follows: (4)

1. Abbreviations used in this paper: $2 \mathrm{DG}, 2$-deoxyglucose; $\mathrm{Fe}_{\mathrm{K}}$, fractional excretion of potassium; GFR, glomerular filtration rate; MFAA, sodium monofluoroacetate; mTAL, medullary thick ascending loop; $\mathrm{TR}_{\mathrm{Na}}$, tubular reabsorption of sodium. 
MFAA, $5 \mathrm{mM}$, and sodium succinate, 5-8 $\mathrm{mM}(n=5)$; (5) MFAA, 5 $\mathrm{mM}$, and sodium alpha ketoglutarate, $5-8 \mathrm{mM}(n=4)$. All chemicals were purchased from Sigma Chemical Co.

(E) Blockade of both aerobic and anaerobic metabolism $(n=11)$. To examine the effect of simultaneous inhibition of mitochondrial respiration and anaerobic glycolysis, kidneys were perfused with the following combinations: (1) rotenone, $10^{-5} \mathrm{M}$ and $2 \mathrm{DG}, 50 \mathrm{mM}(n=6)$; (2) antimycin, $10^{-5} \mathrm{M}$ and $2 \mathrm{DG}, 50 \mathrm{mM}(n=5)$.

\section{Monitoring of renal function and $\mathrm{QO}_{2}$}

Glomerular filtration rate (GFR) was estimated by clearance of ${ }^{14} \mathrm{C}$-inulin. Tubular sodium reabsorption $\left(\mathrm{TR}_{\mathrm{Na}}\right)$ and fractional potassium excretion $\left(\mathrm{Fe}_{\mathrm{K}}\right)$ were expressed as percent filtered load. Because of gross tubular injury, backleak of inulin might have occurred in the experimental groups. These results should therefore be viewed as apparent GFR, $\mathrm{TR}_{\mathrm{Na}}$, and $\mathrm{Fe}_{\mathrm{K}}$. Oxygen uptake $\left(\mathrm{QO}_{2}\right)$ was measured by the Fick method as previously described (7).

\section{Isolated cell experiments}

To test the efficacy of the mitochondrial inhibitors used on the medullary thick ascending limb (mTAL), a limited number of experiments were performed on isolated thick ascending limb cells prepared from the rabbit kidney as previously described (8). Such experiments are difficult to do in the rat because of technical problems in obtaining viable separated tubules or cells and because of the amount of parenchyma needed. As shown in Fig. $1, \mathrm{QO}_{2}$ by the isolated mTAL cells was indeed markedly reduced by the mitochondrial inhibitors, which were used at the same concentration as in the perfused kidney in group $\mathrm{C}$.

\section{Determination of ATP content in renal tissue}

Tissue ATP levels were measured in separate experiments, using 59 kidneys perfused for $20 \mathrm{~min}$ and frozen by immersion in liquid nitrogen. The cortex and entire medulla were separated by free hand dissection of the frozen tissue, on dry ice, in a cold room. The pieces of tissue were weighed out, pulverized in precooled mortars with repeated additions of liquid nitrogen, then rapidly mixed with $6 \%$ perchloric acid and homogenized. After an aliquot was taken for protein measurement, the homogenate was centrifuged and the supernatant neutralized with $\mathrm{K}_{2} \mathrm{CO}_{3}$ and recentrifuged. The final supernatant was frozen until analysis, which was usually within $24 \mathrm{~h}$. The assay for ATP was the enzymatic assay described by Lamprecht and Trautschold (9), who used chemicals purchased from Boehringer Mannheim Biochemicals (Indianapolis, IN). Protein was determined by Lowry's method (10). Levels of ATP obtained in freeze-clamped kidneys taken from anesthetized rats averaged $1.88 \pm 0.16(\mathrm{SD}) \mu \mathrm{mol} / 100 \mathrm{mg}$ protein $(n=17)$, which was comparable to values reported by others $(11,12)$. Values obtained for freeze-clamped isolated perfused kidneys (see Fig. 2) were also comparable to values reported by others (13). ATP levels expressed per $100 \mathrm{mg}$ protein in perfused kidneys tend to be lower when the freeze-clamp technique is used, when compared with frozen dissected tissue, probably because of the substantial amount of albumin-rich perfusate included when the perfused kidney is freeze clamped.

Most ATP determinations were performed on kidneys perfused for

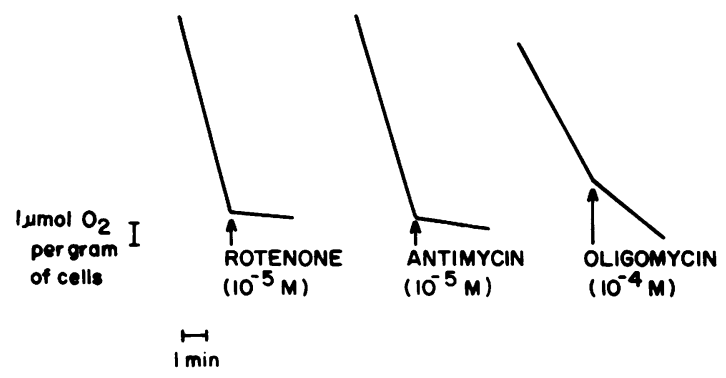

Figure 1. Effects of mitochondrial inhibitors on $\mathrm{QO}_{2}$ by fresh mTAL cells isolated from the rabbit kidney.
$20 \mathrm{~min}$ in order to measure an early parameter of metabolic inhibition rather than a possible loss of cellular ATP secondary to late injury. For three groups $\left(A, C_{(1)}, D_{(3)}\right)$, measurements of tissue ATP made at 90 min as well as at $20 \mathrm{~min}$ of perfusion showed comparable reductions in ATP levels. The data reported in the Results section are those obtained at 20 min of perfusion. The numbers of kidneys perfused in each group for ATP determinations were as follows: group A: 9; group B: 6,4; group C: $6,4,5$; group D: 6,5,4; group E: 5,5.

\section{Morphologic techniques}

The morphology of all kidneys in each group was examined. A threeway stopcock was incorporated into the circuit $5 \mathrm{~cm}$ from the arterial cannula to allow perfusion with the fixation solution, at the same pressure applied during the functional study, for an additional 5-8 min. The fixative solution contained $1.25 \%$ glutaraldehyde in $0.1 \mathrm{M}$ phosphate buffer ( $\mathrm{pH} 7.4$ ). The sections were postfixed in buffered $2 \% \mathrm{OsO}_{4}$, dehydrated and embedded in an araldite-epon 812 mixture. Large $(3 \times 3$ $\mathrm{mm}$ ) sections were cut, containing the cortex and outer stripe (for the evaluation of the proximal tubule) or the outer medulla (for the evaluation of the mTAL). The sections were examined by light microscopy and selected blocks were evaluated by electron microscopy. Analysis of injury was completed in a blinded fashion by Dr. Shanley for the proximal tubule, and by Dr. Rosen for the medullary thick ascending limb.

Quantification of injury in the proximal tubule was performed as follows: For $S_{1}$ and $S_{2}$, the slides were moved by a mechanical stage along a line through the midportion of the cortex, and all tubules included in a high power field $(\times 40)$ were evaluated for the presence or absence of the characteristic $S_{1} / S_{2}$ lesion of hypoxia (brush border clubbing [by electron microscopy, this clubbing lesion was found to represent marked brush border disorganization] and high grade mitochondrial swelling) $(14,15)$. Partially involved tubules were counted as one-half. Tubules with cells having only nuclear changes or apical microvesicles were grouped with normal tubules in the category of minimal lesions. Similarly, for $S_{3}$, tubules were evaluated along a line through the midportion of the outer stripe for the presence of cytoplasmic edema or cell fragmentation, which were characteristic $S_{3}$ lesions of hypoxia $(14,15)$. Lesions other than these did not occur to any significant extent.

The quantification of injury to the mTAL was performed as previously described (5). The damage to mTAL in the inner stripe of the outer medulla was evaluated in three regions: outer (A), mid (B), and inner (C). A percentage score was used to indicate the fraction of tubules involved with minimal to mild (chromatin margination, minor degrees of mitochondrial swelling), moderate (blatant mitochondrial swelling with limited nuclear pyknosis), or severe (blatant mitochondrial swelling with extensive nuclear pyknosis and cell fragmentation) changes.

\section{Statistical analysis}

Results are presented as mean \pm SE. $t$ test was generally used for comparison of different groups. Analysis of variance and Newman-Keul's multiple comparison procedure were used for the data presented in Table I. Linear and curvilinear correlations were analyzed with help from the Division of Computer Medicine.

\section{Results}

Renal function (Table I). In comparison with control perfusions (group A), all inhibitors of mitochondrial respiration (group C) led to marked reductions in GFR, sodium reabsorption, and $\mathrm{QO}_{2}$ in perfused kidneys, which were similar in general to the changes induced by hypoxia and cyanide (group B). The effects of the inhibitors of intermediary metabolism (group D) were comparable in magnitude to the changes observed in groups B and $\mathrm{C}$, although less pronounced, especially with 2DG.

ATP levels. As shown in Figs. 2 and 3, ATP contents of cortex and medulla were significantly depressed by inhibitors of mitochondrial respiration or intermediary metabolism. Blockade 
Table I. Functional Data of Isolated Perfused Kidneys Under Control and Experimental Conditions*

\begin{tabular}{|c|c|c|c|c|c|}
\hline & GFR & $\mathrm{TR}_{\mathrm{N}_{0}}$ & $\mathrm{Fe}_{\mathbf{k}}$ & Perfusion flow & $\mathrm{QO}_{2}$ \\
\hline & $\mathrm{ml} / \mathrm{min}$ & $\%$ & $\%$ & $\mathrm{ml} / \mathrm{min}$ & $\mu \mathrm{mol} / \mathrm{min}$ \\
\hline A Controls $(n=17)$ & $0.50 \pm 0.04 \ddagger$ & $93.1 \pm 0.8 \ddagger$ & $79.0 \pm 6.7$ & $51.3 \pm 1.8^{* *}$ & $5.5 \pm 0.4 \ddagger$ \\
\hline B 1 Hypoxia $(n=7)$ & $0.10 \pm 0.01$ & $41.2 \pm 2.1$ & $78.3 \pm 1.5$ & $31.9 \pm 2.7$ & $0.6 \pm 0.1$ \\
\hline $2 \mathrm{KCN}(n=5)$ & $0.23 \pm 0.08$ & $50.7 \pm 16.0$ & $72.1 \pm 4.2$ & $43.4 \pm 1.8$ & $1.9 \pm 0.7 \ddagger \ddagger$ \\
\hline C 1 Rotenone $(n=6)$ & $0.13 \pm 0.01$ & $31.8 \pm 2.1$ & $83.4 \pm 5.3$ & $33.4 \pm 3.1$ & $0.3 \pm 0.1$ \\
\hline 2 Antimycin $(n=6)$ & $0.22 \pm 0.05$ & $27.6 \pm 2.9$ & $91.0 \pm 6.4$ & $37.7 \pm 4.4$ & $0.5 \pm 0.1$ \\
\hline 3 Oligomycin $(n=7)$ & $0.14 \pm 0.04$ & $31.7 \pm 7.9$ & $67.2 \pm 8.2$ & $31.9 \pm 2.7$ & $0.5 \pm 0.1$ \\
\hline D 1 Monofluoroacetate $(n=6)$ & $0.27 \pm 0.04$ & $62.6 \pm 3.4 \S$ & $54.0 \pm 4.9$ & $34.4 \pm 2.3$ & $1.7 \pm 0.3$ \\
\hline 2 Malonate $(n=5)$ & $0.15 \pm 0.02$ & $40.8 \pm 2.3$ & $71.8 \pm 8.6$ & $36.3 \pm 1.7$ & $1.5 \pm 0.2$ \\
\hline 3 2-deoxyglucose $(n=10)$ & $0.30 \pm 0.03$ & $68.1 \pm 5.5^{\prime \prime}$ & $59.4 \pm 6.2$ & $30.7 \pm 1.1$ & $2.2 \pm 0.2 \S \S$ \\
\hline E 1 Rotenone plus 2DG $(n=6)$ & $0.09 \pm 0.01$ & $4.8 \pm 2.1 \pi$ & $96.5 \pm 2.5$ & $29.3 \pm 2.3$ & $0.1 \pm 0.02$ \\
\hline 2 Antimycin plus 2DG $(n=5)$ & $0.10 \pm 0.01$ & $5.2 \pm 2.0 \pi$ & $97.0 \pm 1.6$ & $33.1 \pm 1.9$ & $0.3 \pm 0.1$ \\
\hline
\end{tabular}

* Data are at $60^{\prime}$ of perfusion and expressed per kidney. $\ddagger P<0.001$ vs. all others. $\S P<0.05$ vs. all others except B2 and D3. " $P<0.05$ vs. all others except D1. I $P<0.01$ vs. all others except $\mathrm{E}$. ${ }^{* *} P<0.01$ vs. all others except $\mathrm{B} 2$. $\quad \ddagger \quad P<0.05$ vs. $\mathrm{Cl}$ and $\mathrm{E} 2$. $\$ \S P<0.05$ vs. all others except B2 and D.

of mitochondrial respiration with rotenone, antimycin, or oligomycin depleted ATP levels more efficiently in the cortex (averaging $67 \%$ reduction from control) than in the medulla $(48 \%$ reduction from control, $P<0.01$ vs. cortex). The reverse was true when anaerobic glycolysis was reduced with $2 \mathrm{DG}(23 \%$ depletion in the cortex vs. $40 \%$ depletion in the medulla, $P<0.01$ ). The combination of rotenone or antimycin with 2DG (group E) produced the most profound reductions of ATP levels in both cortex and medulla: 72 vs. $67 \%$ depletion, respectively (not significantly different from each other).

Morphological changes: proximal tubule $\left(S_{1}, S_{2}\right)$. Morphological evaluation of the proximal tubule showed that ATP depletion produced marked structural alterations regardless of the way in which it was attained. In the first segments of the proximal tubule $\left(S_{1}\right.$ and $\left.S_{2}\right)$, clubbing of microvilli in the brush border and mitochondrial swelling were produced by hypoxia (Fig. 4 $A$ ) and cyanide (Fig. 4 B). Qualitatively identical changes were observed after inhibition of intermediary metabolism (Fig. $4 C$ )

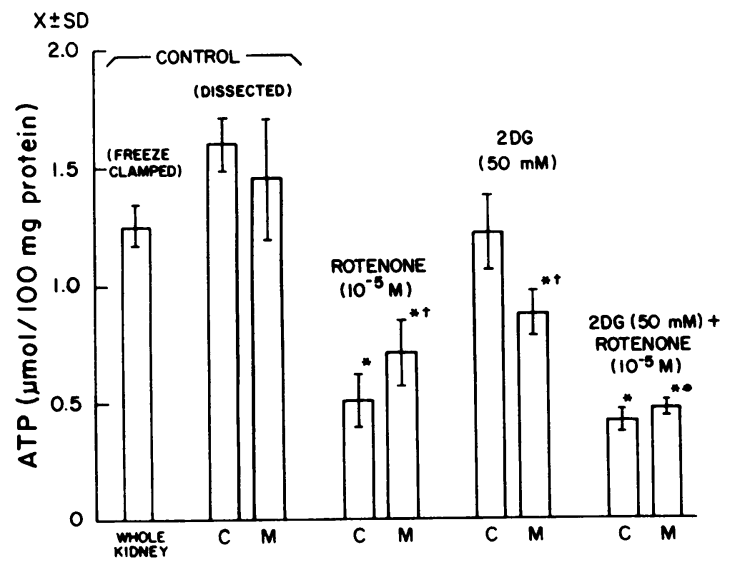

Figure 2. ATP levels of cortex (C) and medulla (M) under control conditions and metabolic inhibition. ${ }^{*} P<0.001$ vs. control. $\dagger P$ $<0.02$ vs. cortex. $\bullet P<0.001$ vs. rotenone or $2 D G$ alone. or mitochondrial respiration (Fig. 4, $D$ and $E$ ). Combination of 2DG with an inhibitor of mitochondrial respiration (group E) led to extension of these hypoxiclike lesions to virtually all proximal tubules. The extent of these changes was found to be inversely correlated with the cortical ATP content measured in parallel experiments (see Fig. $3 A$ ).

Morphological changes: proximal tubule $\left(S_{3}\right)$. In the last segment of the proximal tubule $\left(S_{3}\right)$, hypoxic lesions (observed in group B) consisted of cytoplasmic edema or cell fragmentation (Fig. 5). These lesions were not reproduced to any significant extent by the inhibitors of intermediary metabolism (group D; monofluoroacetate, malonate, or 2DG). Similar lesions were inconsistently reproduced by cyanide and by the inhibitors of mitochondrial respiration (group C), until more severe ATP depletion was reached by combining rotenone or antimycin with 2DG (Fig. 6). The extent of these changes was found to be inversely correlated with the medullary ATP content (Fig. 7).

Morphological changes: $m T A L$. Morphological evaluation of the mTAL showed divergent results, depending on the means used for ATP depletion (Fig. 3 B). Hypoxia and cyanide (group $B)$ intensified the anoxic damage regularly observed in controls $(5,16)$, as illustrated in Fig. 8. By contrast, inhibition of mitochondrial respiration (group $\mathrm{C}$ ) or of intermediary metabolism (group D) produced only minimal alterations in the mTAL, as illustrated in Fig. 9, $A-D$. The combination of 2DG with a mitochondrial inhibitor (group E) led to the appearance of cytoplasmic edema and chromatin margination (Fig. 9, $E$ and $F$ ) but without cell fragmentation or nuclear pyknosis, lesions that are characteristic of mTAL hypoxia $(5,16,17)$, as represented in Fig. 8. In fact, in all these experimental groups (C-E), metabolic inhibition significantly decreased the proportion of mTALS, demonstrating moderate to severe damage, as illustrated in Fig. $3 B$. Detailed quantification of the injury to mTAL under these conditions is given in Fig. 10.

Reversal of proximal tubule and $m T A L$ injury. Reversal of monofluoroacetate inhibition with succinate or alpha ketoglutarate was indicated by a significant increase in $\mathrm{QO}_{2}$ (Fig. 11). In the proximal tubule, both succinate and alpha ketoglutarate 

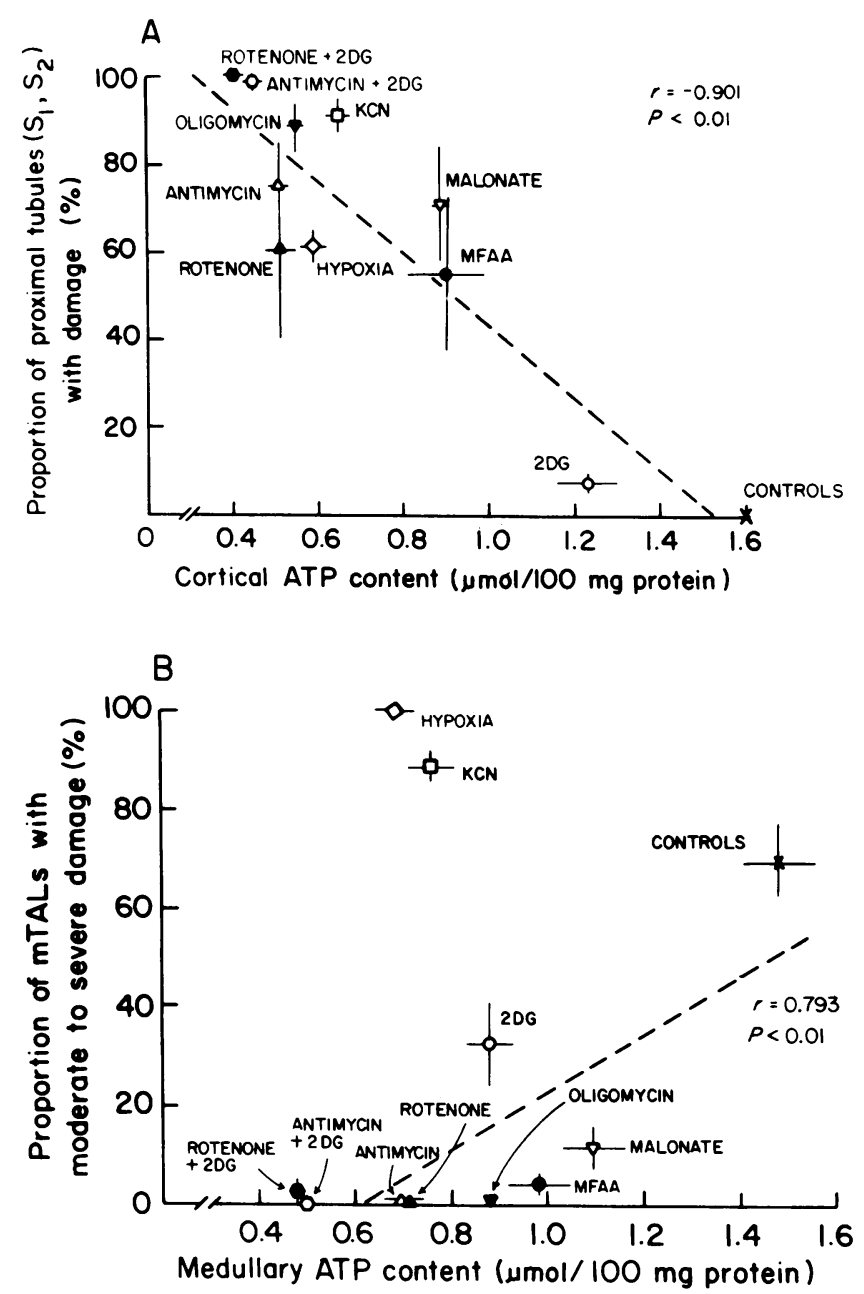

Figure 3. $(A)$ Correlations between tissue ATP content and histological damage in the proximal tubule $\left(S_{1}, S_{2}\right)$. The lesions characteristic of hypoxic damage in the proximal tubule were brush border clubbing and high grade mitochondrial swelling (see Methods and Fig. 4). (B) Correlation between tissue ATP content and histological damage in the mTAL. The hypoxic lesions observed in the mTAL include mitochondrial swelling, nuclear pyknosis, and cytoplasmic disruption as described previously (5) and illustrated in Fig. 8. The quantitation shown is the average for areas B and C (see Methods and Fig. 10).

tended to restore cell integrity. In the mTAL, on the other hand, alpha ketoglutarate tended to reestablish the anoxic injury, as did succinate, but to a smaller extent (Fig. 11).

Comparison of tissue ATP levels during hypoxia, mitochondrial inhibition, and hypoxia with ouabain treatment. Renal cortical and medullary ATP levels during hypoxia, inhibition of mitochondrial respiration with rotenone or antimycin, and inhibition of active transport with ouabain in hypoxia are shown in Fig. 12. ATP levels in the presence of rotenone or antimycin were not significantly different from those measured with hypoxia alone, and were significantly lower than those measured with hypoxia in the presence of ouabain.

\section{Discussion}

Depletion of cellular energy stores is generally viewed as an important if not crucial consequence of anoxia that results in cell swelling, calcium influx, and membrane disruption (1-3). It is therefore not surprising that ATP depletion achieved by inhibitors of intermediary metabolism or mitochondrial respiration reproduced cellular damage morphologically identical to that elicited by hypoxia or cyanide in the proximal tubule. In these experiments, we measured ATP levels in whole cortex. To the extent that the proximal tubule forms a major part of the cellular mass in the cortex, depletion of cortical ATP reflects reduced energy stores in this nephron segment. Rotenone and antimycin $\left(10^{-5}-10^{-6} \mathrm{M}\right)$ have in fact been shown to deplete markedly the ATP content of isolated proximal tubules to $\sim 10-20 \%$ of controls $(18,19)$. The type of morphological response to ATP depletion in the first segments of the proximal tubule $\left(S_{1}\right.$ and $\left.S_{2}\right)$ was similar regardless of the agent used, and consisted of clubbing of the microvilli and mitochondrial swelling. The magnitude of these changes was proportional to the degree of ATP depletion. When inhibition of aerobic metabolism was only partial (as with monofluoroacetate), as indicated by residual $\mathrm{QO}_{2}$ and sodium reabsorption (Table I) and by only moderate degrees of ATP depletion, lesions were observed in a moderate number of tubules. Maximal ATP depletion with either rotenone or antimycin plus 2DG resulted in the involvement of all $\mathrm{S}_{1}$ and $\mathrm{S}_{2}$ segments examined (Fig. $3 \mathrm{~A}$ ). The relative morphological refractoriness of the proximal tubule to 2DG is consistent with its lack of effect on cortical ATP and the reported poor utilization of glucose for either aerobic or anaerobic metabolism in this nephron segment $(4,20)$.

The importance of ATP synthesis via the citric acid cycle in the proximal tubule is underlined by the experiments with monofluoroacetate. Damage to the proximal tubule produced by this inhibitor of citrate oxidation was attenuated by succinate and alpha ketoglutarate, which enter the Krebs cycle after aconitase and are readily metabolized by proximal tubular cells (19, 21). These substrates bypassed the metabolic block of monofluoroacetate as indicated by an increase in $\mathrm{QO}_{2}$, and significantly reduced the severity of cellular damage (Fig. 11). The close correlation between the morphological changes induced in the proximal tubule by oxygen deprivation and inhibition of aerobic metabolism indicate that decreased energy supply in this nephron segment is the probable cause of hypoxic injury to these cells.

In contrast to the proximal tubule, the response of the mTAL was paradoxical, since anoxic injury could not be reproduced by metabolic inhibition. It should be pointed out that ATP levels in the whole medulla, as measured, are not an accurate measure of energy stores in the mTAL, since this segment constitutes only a small fraction of the entire medulla (22). Medullary ATP content may, however, be regarded as an indication of the intensity of inhibition of energy metabolism in a portion of renal tissue known to have a large capacity for anaerobic metabolism (23). Intrinsic resistance of the mTAL cells to the various inhibitors appears unlikely since, in the isolated thick ascending limb cells of the rabbit kidney, various mitochondrial inhibitors effectively suppressed $\mathrm{QO}_{2}$ (Fig. 1) as they do in proximal tubules (18). The mitochondrial inhibitors, however, depleted ATP content to a lesser extent in the medulla than in the cortex, as predicted from the greater reserve for anaerobic glycolysis in the medulla (23) and in the mTAL in particular (24). Indeed, the combination of $2 \mathrm{DG}$ with rotenone or antimycin led to a significant further depletion of the medullary ATP, to levels as low as those measured in the cortex (Figs. 2 and 3). Surprisingly, even under these conditions, the characteristic severe hypoxic lesions described in the mTAL, i.e., nuclear pyknosis and cell fragmentation, could not be reproduced. Only the inhibition of 


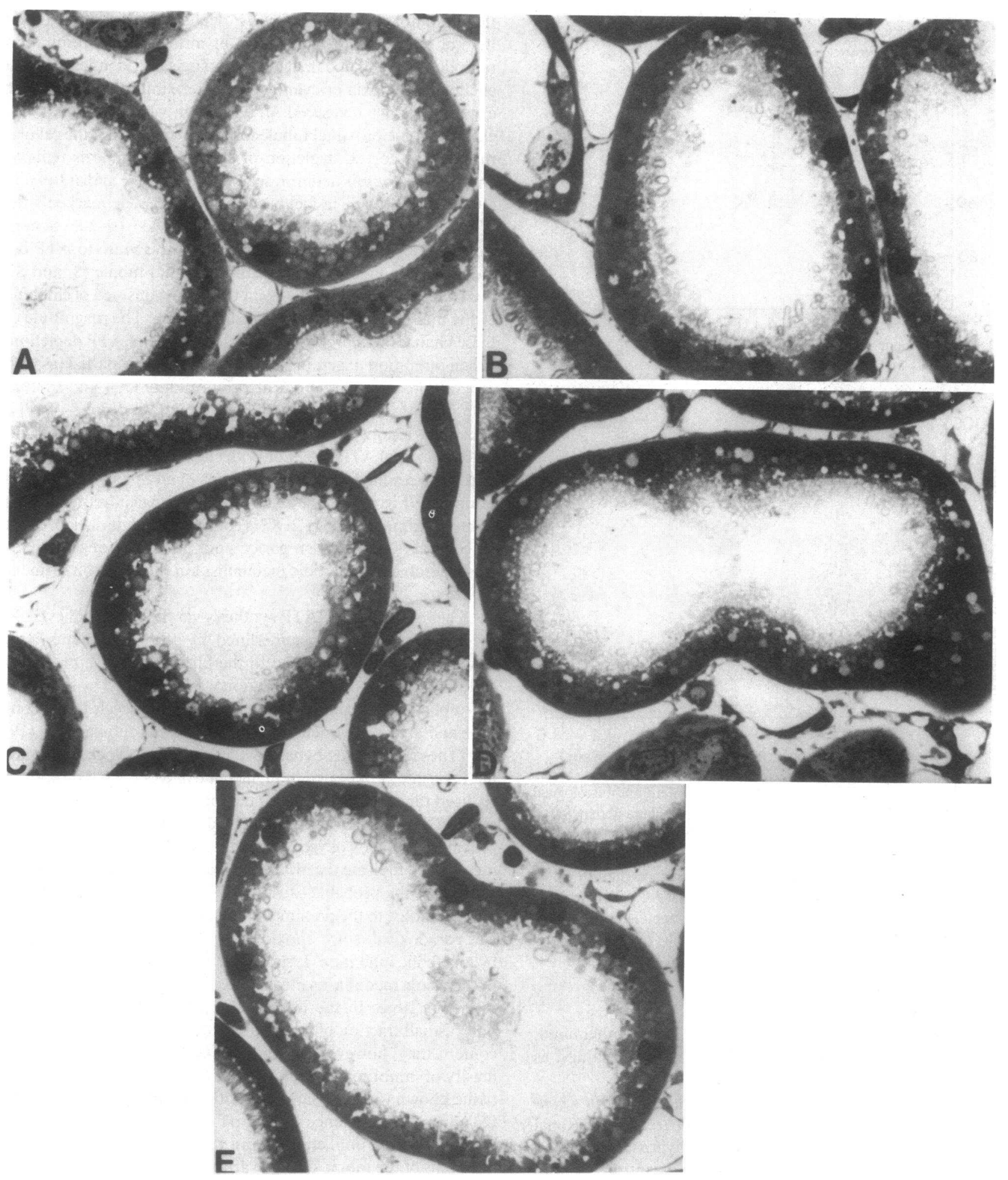

Figure 4. Morphology of proximal tubule $\left(S_{1}, S_{2}\right)$ in hypoxia and during metabolic inhibition: mitochondrial swelling and brush border clubbing are lesions characteristic of hypoxia $(A)$. Similar lesions occur under conditions of histotoxic anoxia, $\mathrm{KCN}(B)$, or injury produced

the final step of respiration (electron transfer to oxygen at cytochrome $a, a_{3}$ ) by hypoxia or cyanide generated severe injury. More proximal inhibition along the mitochondrial electron chain, or at the level of substrate oxidation, did not. Thus, this mode of injury appears to depend on continued mitochondrial by inhibitors of intermediary metabolism, MFAA $(C)$, and mitochondrial electron transport, such as oligomycin $(D)$ and antimycin $(E) . \times$ 880 .

electron flow in the face of limited oxygen, a situation that might conceivably lead to the production of electron-dependent free radicals (25). It should be noted that under certain conditions, antimycin (26) and rotenone (27) can decrease the formation of oxygen-free radicals by mitochondria. In addition, the un- 


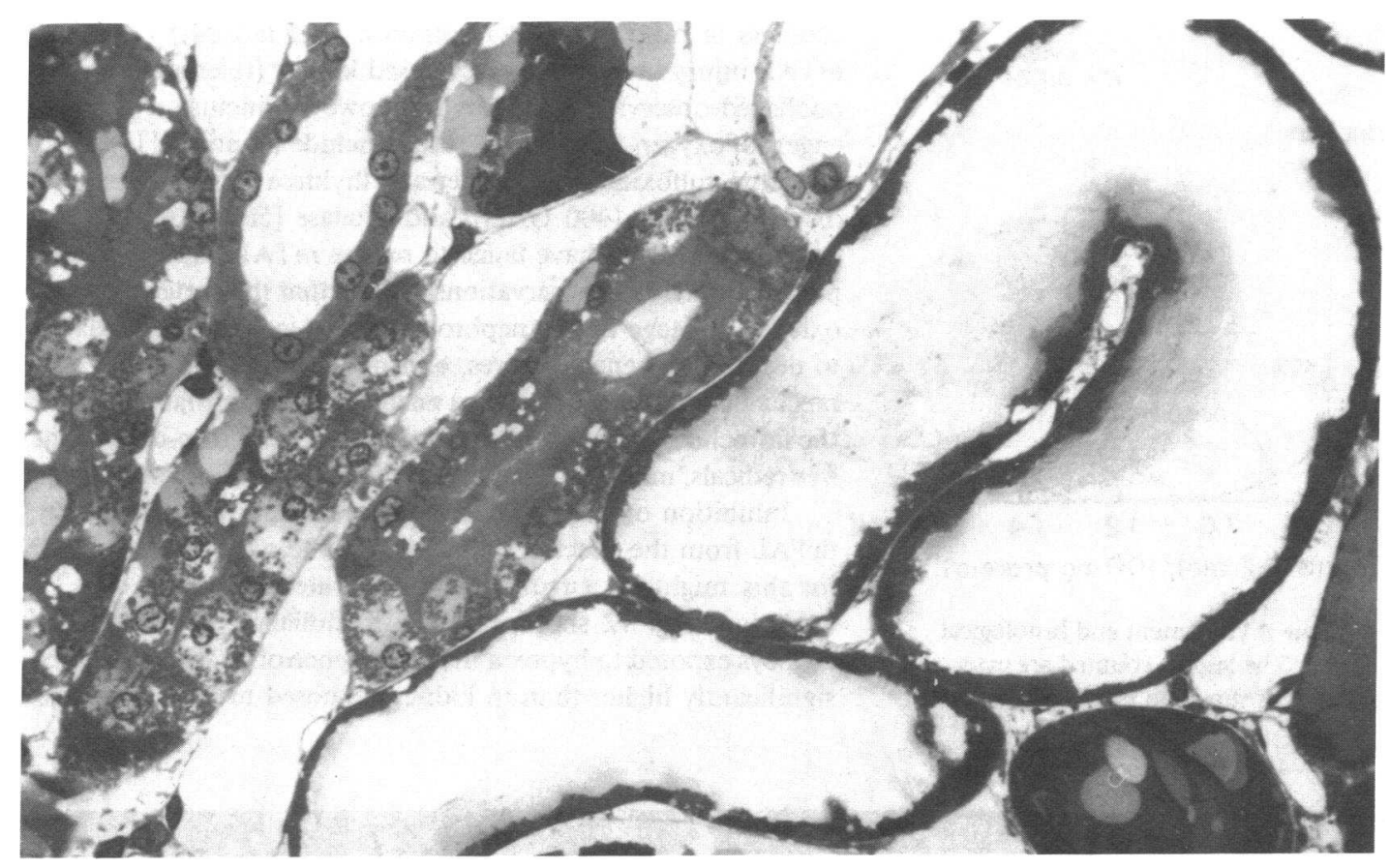

Figure 5. Morphology of the proximal tubule $\left(\mathrm{S}_{3}\right)$ in hypoxia. Under hypoxic conditions, the $\mathrm{S}_{3}$ segment of the PT responds with two overt types of cell injury: cell fragmentation with tubular dilatation (right), or marked cellular edema with luminal occlusion $(l e f t)$. $\times 430$.

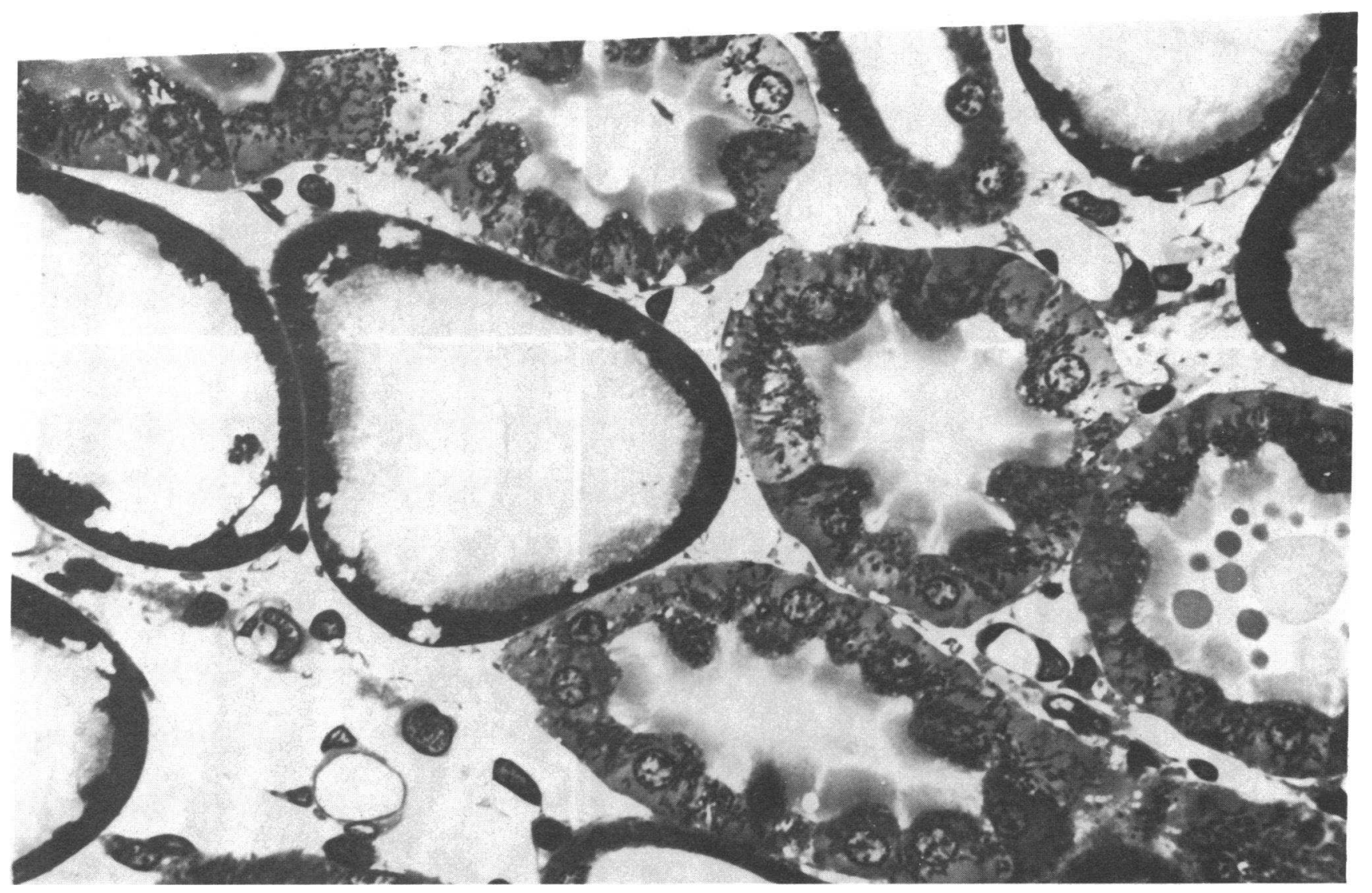

Figure 6. Morphology of the proximal tubule $\left(\mathrm{S}_{3}\right)$ with combined mitochondrial blockade (antimycin) and inhibition of glycolysis (2DG). Some tubules show cytoplasmic edema (right), while others show cell fragmentation $(l e f t)$, lesions similar to those seen under hypoxic conditions (see Fig. 5). $\times 800$. 


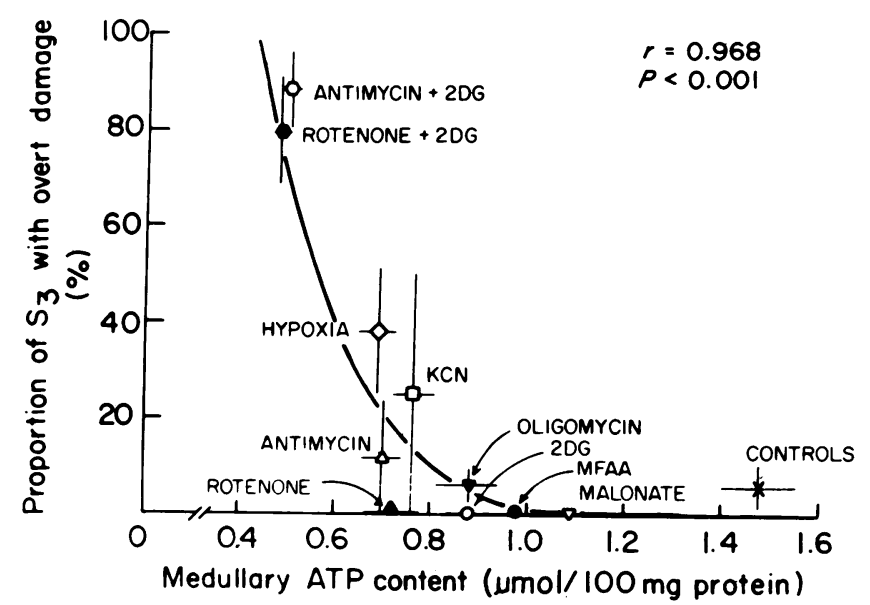

Figure 7. Correlations between tissue ATP content and histological damage in the proximal tubule $\left(\mathbf{S}_{3}\right)$. The lesions counted are cytoplasmic edema or cell fragmentation as shown in Fig. 5 (see Methods). couplers of mitochondrial respiration tend indeed to increase mTAL injury in the isolated perfused kidney (reference 16; unpublished observations). Thus far, however, inclusion of scavengers of oxygen-free radicals (these include mannitol [10 $\mathrm{mM}$ ], dimethyl sulfoxide [0.3 M], tetramethylurea [ $25 \mathrm{mM}$ ], superoxide dismutase $[400 \mathrm{U} / \mathrm{ml}]$, and catalase $[500 \mathrm{U} / \mathrm{ml}]$ ) in the perfusion medium have failed to reduce mTAL damage in this preparation. These observations suggest that the pattern of hypoxic cell damage in this nephron segment is not simply related to depletion of energy stores, and may result from a different mechanism of injury. Aberrant energy biotransformation within the mitochondria, such as the production of electron-dependent free radicals, may be involved, although this remains conjectural.

Inhibition of transport activity by ouabain can protect the mTAL from the effect of hypoxia (16), and one possible reason for this might be a reduction in the rate and extent of ATP depletion. Fig. 12 shows that the medullary ATP content of kidneys exposed to hypoxia in the presence of ouabain is indeed significantly higher than in kidneys exposed to hypoxia alone.

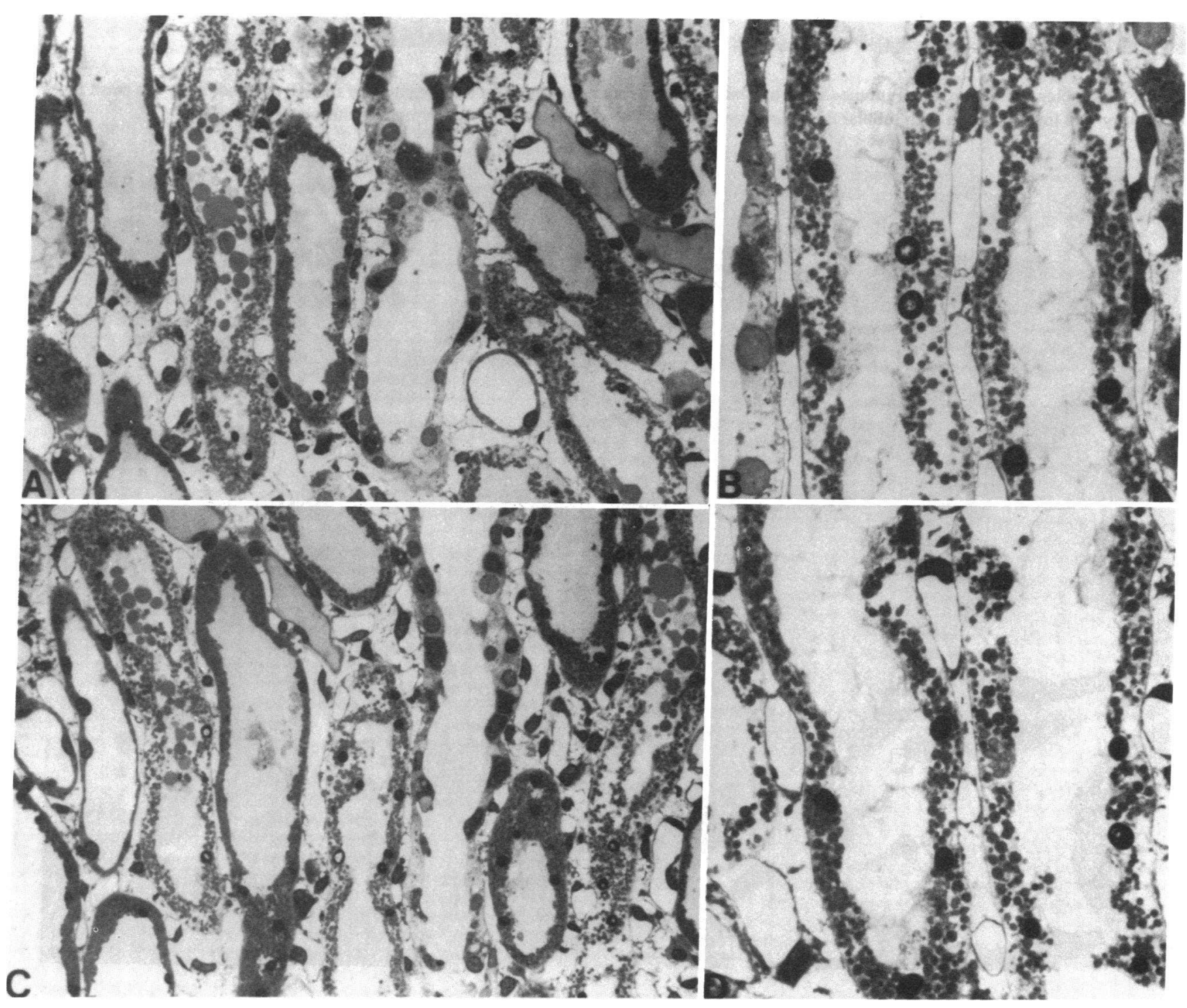

Figure 8. Morphology of the mTAL in hypoxia and histotoxic anoxia. The extensive destruction of the mTAL that occurs under hypoxic conditions $(A$ and $B)$ is reproduced when $\mathrm{KCN}(>1.0 \mathrm{mM})$ is included in the perfusate $(C$ and $D) . \times 350 ; \times 880$. 


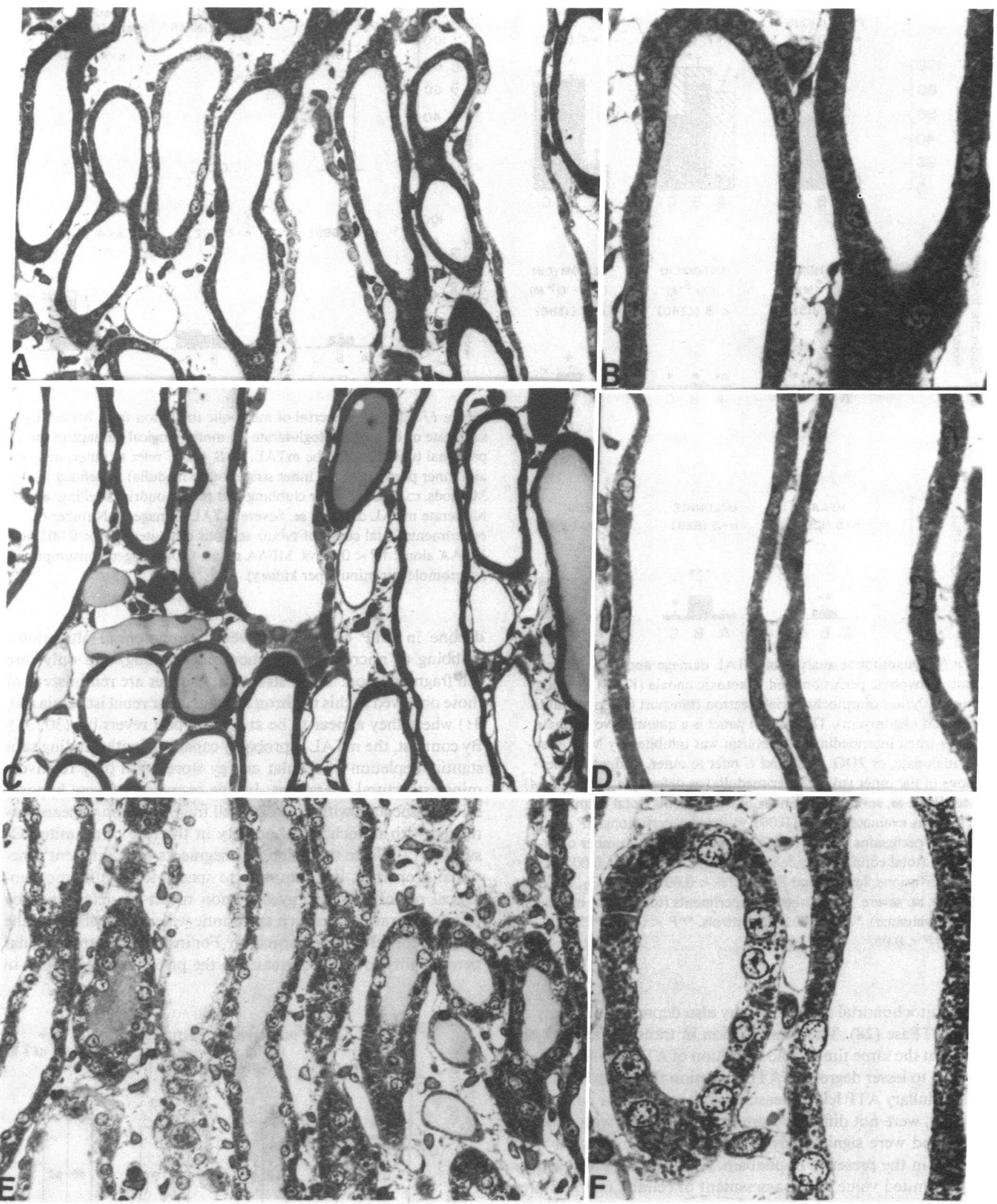

Figure 9. Morphology of the mTAL during metabolic inhibition. In marked contrast to the proximal tubule, inhibition of intermediary metabolism with MFAA ( $A$ and $B$ ) or mitochondrial electron transport with rotenone $(C$ and $D)$ produces minimal alterations in mTAL. Only when both anaerobic glycolysis (2DG) and respiratory chain inhibition is present (antimycin) do changes occur. These changes are chromatin margination and cellular edema, without obvious mitochondrial swelling ( $E$ and $F$ ). By our criteria (described in Methods), these alterations are defined as mild injury. Even under these circumstances, cellular integrity is relatively intact when compared to hypoxic injury (see Fig. $8, A$ and $B$ ). $\times 350 ; \times 880$. 

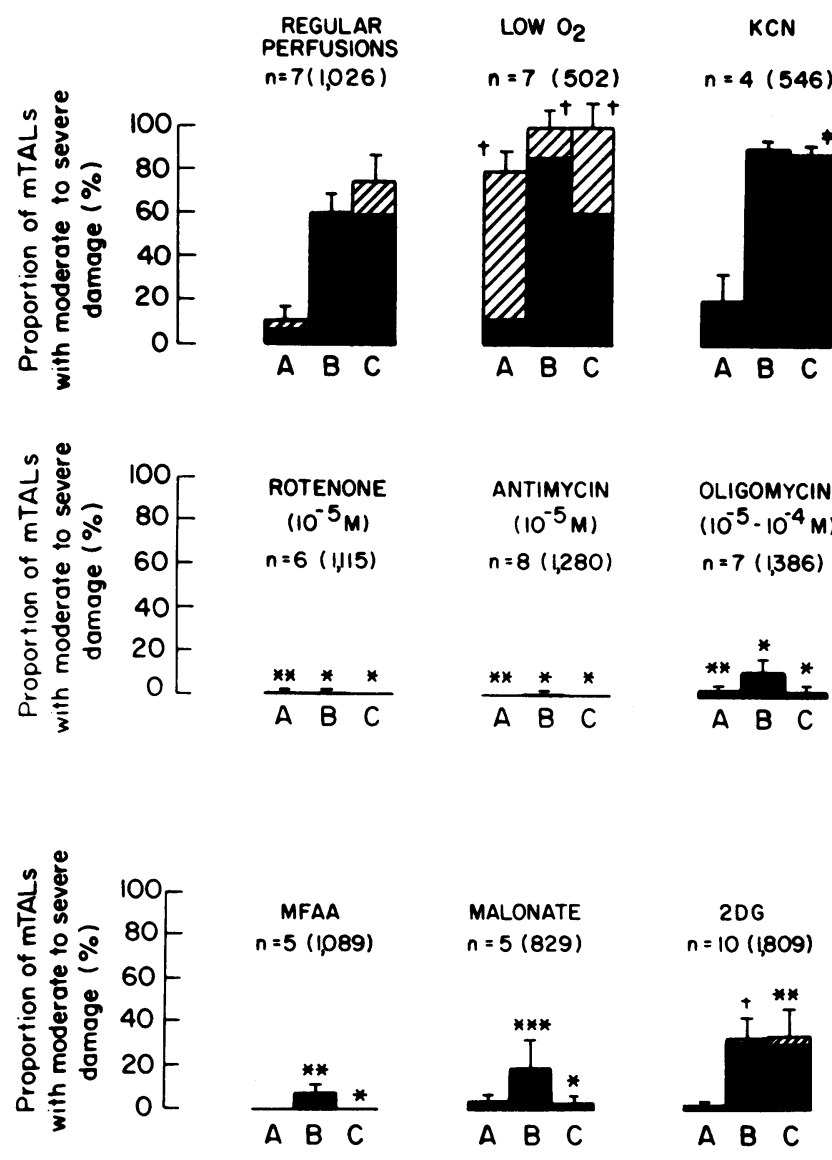

Figure 10. Quantitative analysis of mTAL damage under conditions of control, hypoxic perfusion, and histotoxic anoxia $(\mathrm{KCN})$ as well as during inhibition of mitochondrial electron transport (rotenone, antimycin, and oligomycin). The bottom panel is a quantitative estimate of injury when intermediary metabolism was inhibited by MFAA, sodium malonate, or 2DG. $A, B$, and $C$ refer to outer, mid, and inner portions of the inner stripe (outer medulla) as defined in Methods. $(A)$ -, Moderate; $\boldsymbol{n}$, severe. $n$, Number of experiments (total count of tubule sections evaluated). $† P<0.001$ vs. regular perfusions. $¥ P<0.05$ vs. regular perfusions. $(B) \square$, Moderate; $\square$, severe. $n$, Number of experiments (total count of tubule sections evaluated). ${ }^{*} P<0.001$ vs. regular perfusions, low $\mathrm{O}_{2}$ and $\mathrm{KCN}$. ${ }^{* *} P<0.001$ vs. low $\mathrm{O}_{2}$. $(C)=$, Moderate; $m$, severe. $n$, Number of experiments (total count of tubule sections evaluated). ${ }^{*} P<0.001$ vs. controls. ${ }^{* *} P<0.005$. ${ }^{* * *} P$ $<0.02$. $+P<0.05$.

Some mitochondrial inhibitors may also depress the activity of Na-K-ATPase (28). Thus, a reduction in transport activity occurring at the same time as the inhibition of ATP synthesis might have led to lesser degrees of ATP depletion than hypoxia alone. The medullary ATP levels measured with rotenone or antimycin, however, were not different from those measured with hypoxia alone, and were significantly lower than those measured with hypoxia in the presence of ouabain. Although ATP levels may have a limited value in the assessment of cellular energy stores (29), in the present study the low levels obtained with metabolic and mitochondrial blockers (or their combination) can reasonably be assumed to represent inhibition of energy metabolism and reduction of the energy available for cellular functions.

The remarkable difference in the response of the proximal tubule and the mTAL to ATP depletion is of particular interest. The proximal tubule $\left(S_{1}, S_{2}\right)$ appears to express even a moderate

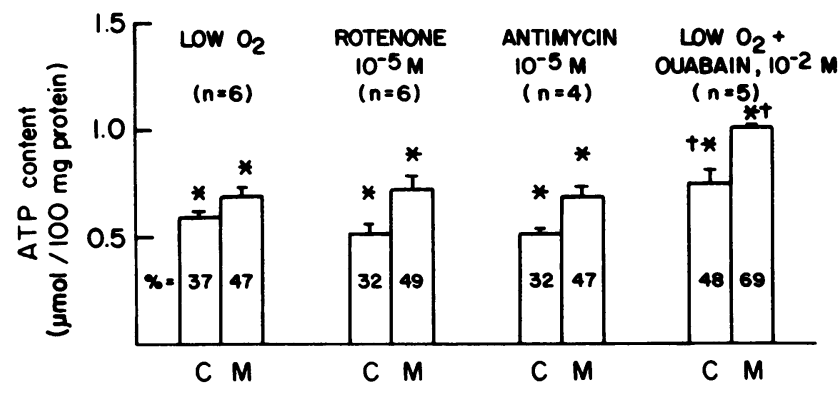

Figure 12. Comparison of tissue ATP levels (cortex, C; medulla, M) when perfused kidneys were exposed to low $\mathrm{O}_{2}$, rotenone, antimycin, or low $\mathrm{O}_{2}$ plus ouabain. The numbers inside the bars indicate the percentage of control ATP levels. C, Cortex; $M$, medulla. ${ }^{*} P<0.001$ vs. controls. $\dagger P<0.005$ vs. low $\mathrm{O}_{2}$, rotenone or antimycin.

Figure 11. Effects of reversal of metabolic inhibition from MFAA by succinate or by alpha ketoglutarate on morphological damage in the proximal tubule and in the mTAL. $A, B$, and $C$ refer to outer, mid, and inner portions of the inner stripe (outer medulla) as defined in Methods. $\square$, Brush border clubbing and mitochondrial swelling; $\boldsymbol{m}$, Moderate mTAL damage; $a$, Severe mTAL damage. $n$, Number of experiments (total count of tubule sections evaluated). ${ }^{*} P<0.001$ vs. MFAA alone. $\dagger P<0.05$ vs. MFAA alone. $Q_{2}$, oxygen consumption (micromole per minute per kidney).

decline in ATP content by overt morphological alterations: clubbing of microvilli, mitochondrial swelling, and only rare cell fragmentation. These structural changes are reminiscent of those observed in this nephron segment after renal ischemia (30, 31 ) where they appear to be at least in part reversible $(30,31)$. By contrast, the mTAL is probably capable of withstanding substantial depletion of cellular energy stores with only relatively minor structural alterations. In this segment, a severe hypoxic injury associated with extensive cell fragmentation appears correlated with mitochondral activity in the face of a limited $\mathrm{O}_{2}$ supply (17). Since these nephron segments have different functional properties, it is tempting to speculate that the morphological response to energy depletion might be determined by cellular characteristics such as osmotic water permeability or the capacity for gel-sol transformation. For instance, the transtubular permeability to water is greater in the proximal tubule than in 
the mTAL by about three orders of magnitude (32), and seems to derive from a greater transcellular water permeability (32) that is possibly also related to greater membrane fluidity (33). By contrast, the mTAL is characterized by a very low hydraulic conductivity and by the presence of a gel-forming Tamm-Horsfall protein that might conceivably play a role in the stability of these cells to osmotic stresses (34). Preliminary observations using an impermeant solute (polyethylene glycol) added to the perfusate show considerable alterations of cell morphology in the proximal tubule, with little change in thick ascending limbs (35). That inhibition of transport activity (as with ouabain) offers only marginal protection from hypoxia in the proximal tubule (35), while preventing damage in the mTAL, supports the notion of disparate mechanisms for hypoxic cell injury along the nephron. The easily elicited morphological changes associated with abnormal energy metabolism in the proximal tubule could underlie the prominent proximal tubular damage described in various toxic or anoxic nephropathies. The transport-dependent nature of mTAL injury may be important in acute renal failure (36).

The response of the $S_{3}$ segment of the proximal tubule to ATP depletion is of interest because of its reported vulnerability after in vivo ischemia (31) and because it appeared intermediate between the response of the earlier portion of the proximal tubule and that of the mTAL. Comparison of Fig. 7 to Fig. $3 A$ shows that $S_{3}$ was more resistant than $S_{1}$ and $S_{2}$ to procedures producing depletion of cortical tissue ATP. With the addition of 2DG and more advanced ATP depletion, severe cell damage (fragmentation or edema) became extensive. This observation suggests a contribution of anaerobic metabolism to cell integrity in $S_{3}$, despite reports claiming little or no capacity for anaerobic glycolysis in this nephron segment $(4,24)$. It is conceivable that when mitochondrial respiration is blocked, additional inhibition with 2DG of a quantitatively small anaerobic ATP production would induce the marked qualitative changes in cell morphology observed.

In conclusion, the response of various portions of the nephron of the isolated perfused rat kidney to hypoxic or metabolic insults is heterogeneous. Thus, different mechanisms of damage, operating in specific portions of the tubule, may underlie the diverse patterns of injury observed in vivo.

\section{Acknowledgments}

The authors are grateful to Dr. Bernard Ransil of the Core Laboratory of the General Clinical Research Center, Beth Israel Hospital, for his help in the statistical analysis.

This work was supported in part by U. S. Public Health Service grant AM 18078. This work was also supported by grant $84-44$ from the U.S.Israel Binational Science Foundation (BSF), Jerusalem, Israel. Dr. Brezis is a fellow of the Bat-Sheva Foundation.

\section{References}

1. Farber, J. L. 1982. Biology of disease. Membrane injury and calcium homeostasis in the pathogenesis of coagulative necrosis. Lab. Invest. 47:114-121.

2. Chaudry, I. H. 1983. Cellular mechanisms in shock and ischemia and their correction. Am. J. Physiol. 245:R117-R134.

3. Robbins, S. L., R. S. Cotran, and V. Kumar, editors. 1984. Cellular injury and adaptation. Pathologic Basis of Disease. W. B. Saunders \& Co., Philadelphia. 1:8.

4. Guder, W. G., and B. D. Ross. 1984. Enzyme distribution along the nephron. Kidney Int. 26:101-111.
5. Brezis, M., S. Rosen, P. Silva, and F. H. Epstein. 1984. Selective vulnerability of the thick ascending limb to anoxia in the isolated perfused kidney. J. Clin. Invest. 73:182-189.

6. Ross, B. D., F. H. Epstein, and A. Leaf. 1973. Sodium reabsorption in the perfused rat kidney. Am. J. Physiol. 225:1165-1171.

7. Silva, P., R. Hallac, K. Spokes, and F. H. Epstein. 1982. Relationship among gluconeogenesis, $\mathrm{QO}_{2}$, and $\mathrm{Na}+$ transport in the perfused rat kidney. Am. J. Physiol. 242:F508-F513.

8. Eveloff, J. E., E. Bayerdorffer, P. Silva, and R. Kinne. 1981. Sodium chloride transport in the thick ascending limb of Henle's loop. Pfluegers Arch. Eur. J. Physiol. 389:263-270.

9. Lamprecht, W., and I. Trautschold. Determination with hexokinase and glucose-6-phosphate dehydrogenase. 1974. In Methods of Enzymatic Analysis, Vol 4. H. U. Bergmeyer, editor. Academic Press, Inc., New York. 2101-2110.

10. Layne, E. 1957. Spectrophotometric and turbidimetric methods for measuring proteins. In Methods in Enzymology, Vol. III. S. P. Colowick and N. D. Kaplan, editors. Academic Press, Inc., New York. 448-450.

11. Needleman, P., J. V. Passonneau, and O. H. Lowry. 1968. Distribution of glucose and related metabolites in rat kidney. Am. J. Physiol. 215:655-659.

12. Jones, N. F., and L. G. Welt. 1967. Adenosine triphosphate in rat renal papilla: effects of vasopressin and of ischemia. Am. J. Physiol. 212:939-944.

13. Trimble, M. E., and R. H. Bowman. 1973. Renal $\mathrm{Na}^{+}$and $\mathrm{K}^{+}$ transport: effect of glucose, palmitate and alpha-bromopalmitate. Am J. Physiol. 225:1057-1062.

14. Shanley, P., M. Brezis, K. Spokes, P. Silva, F. H. Epstein, and S. Rosen. 1984. Gradients of injury to the proximal tubule in the isolated hypoxic perfused kidney. Clin. Res. 32:457A.

15. Shanley, P., M. Brezis, K. Spokes, P. Silva, F. H. Epstein, and S. Rosen. 1985. Transport-dependent cell injury in the S3 segment of the proximal tubule. Kidney Int. 27:238A.

16. Brezis, M., S. Rosen, K. Spokes, P. Silva, and F. H. Epstein. 1984. Transport-dependent anoxic cell injury in the isolated perfused rat kidney. Am. J. Pathol. 116:327-341.

17. Brezis, M., S. Rosen, P. Shanley, K. Spokes, P. Silva, and F. H. Epstein. 1984. Electron flow dependent cell damage: an unrecognized element of anoxic injury? Clin. Res. 32:442A.

18. Gullans, S. R., P. C. Brazy, S. P. Soltoff, V. W. Dennis, and L. J. Mandel. 1982. Metabolic inhibitors: effects on metabolism and transport in the proximal tubule. Am. J. Physiol. 243:F133-F140.

19. Blumenthal, S. S., R. A. Ware, and J. G. Kleinman. 1983. Sodium gradient-driven transport processes in ATP depleted renal tubules. Am. J. Physiol. 244:C331-C335.

20. Klein, K.L., M.-S. Wang, S. Torikai, W. D. Davidson, and K. Kurokawa. 1981. Substrate oxidation by isolated single nephron segments of the rat. Kidney Int. 20:29-35.

21. Lehninger, A. L. 1970. The tricarboxylic acid cycle and the phosphogluconate pathway. Biochemistry. Worth Publishers, Inc., New York. 16:347.

22. Knepper, M. A., R. A. Danielson, G. M. Saidel, and R. S. Post. 1977. Quantitative analysis of renal medullary anatomy in rats and rabbits. Kidney Int. 12:313-323.

23. Cohen, J. J. 1979. Is the function of the renal papilla coupled exclusively to anaerobic metabolism? Am. J. Physiol. 236:F423-F433.

24. Bagnasco, S., M. Burg, R. Balaban, and D. Good. 1984. Lactic acid production by nephron segments of the rat. Clin. Res. 32:440A.

25. Demopoulos, H. B., E. Flamm, M. Seligmann, and D. D. Pietronigro. 1982. Oxygen free radicals in central nervous system ischemia and trauma. In Pathology of Oxygen. A. P. Autor, editor. Academic Press, Inc., New York. 127-155.

26. Loschen, G., A. Azzi, and L. Flohe. 1974. Mitochondrial $\mathrm{H}_{2} \mathrm{O}_{2}$ formation and energy conservation. In Glutathione. L. Flohe, H. C. H. Benohr, H. Sies, H. D. Waller, and A. Wendel, editors. Georg Thieme Publishers, Stuttgart. 228-236.

27. Gauduel, Y., and M. Duvelleroy. 1984. Direct effects of oxygen 
toxicity during the cardiac "oxygen paradox." In Oxygen Transport to Tissue-VI. D. Bruane, H. Bichet, and D. Reneau, editors. Plenum Press, New York. 451-461.

28. Gyory, A. Z., and R. Kinne. 1971. Energy source for transepithelial sodium transport in rat renal proximal tubules. Pfluegers Arch. Eur. J. Physiol. 327:234-260.

29. Banks, B. E. C., and C. A. Vernon. 1970. Reassessment of the role of ATP in vivo. J. Theor. Biol. 29:301-326.

30. Trump, B. F., I. K. Berezesky, and R. A. Cowley. 1982. The cellular and subcellular characteristics of acute and chronic injury with emphasis on the role of calcium. In Pathophysiology of Shock, Anoxia and Ischemia. R. A. Cowley and B. F. Trump, editors. Williams \& Wilkins Co., Baltimore. 6-46.

31. Venkatachalam, M. A., D. B. Bernard, J. D. Donohoe, and N. G. Levinsky. 1978. Ischemic damage and repair in the rat proximal tubule. Differences among the S1, S2 and S3 segments. Kidney Int. 14: $31-49$.

32. Berry, C. A. 1983. Water permeability and pathways in the proximal tubule. Am. J. Physiol. 245:F279-F294.

33. LeGrimellec, C., S. Carriere, J. Cardinal, and M. C. Giocondi. 1983. Fluidity of brush border and basolateral membranes from human kidney cortex. Am. J. Physiol. 245:F227-F231.

34. Hoyer, J. R., and M. W. Seiler. 1979. Pathophysiology of TammHorsfall protein. Kidney Int. 16:279-289.

35. Brezis, M., S. Rosen, P. Shanley, K. Spokes, P. Silva, and F. H. Epstein. 1984. Disparate mechanisms for anoxic cell injury along the nephron: implications for acute renal failure. IXth Int. Cong. Nephrol., Los Angeles. 321A.

36. Brezis, M., S. Rosen, P. Silva, and F. H. Epstein. 1984. Renal ischemia: a new perspective. Kidney Int. 26:375-383. 